\title{
DOSE-DEPENDENT EFFECTS OF CAPSAICIN ON PRIMARY SENSORY NEURONS IN THE NEONATAL RAT ${ }^{1}$
}

\author{
J. I. NAGY, ${ }^{2}$ L. L. IVERSEN, M. GOEDERT, D. CHAPMAN, AND S. P. HUNT \\ Medical Research Council Neurochemical Pharmacology Unit, Medical Research Council Centre, Hills Road, Cambridge, \\ United Kingdom CB2 2QH
}

Received July 8, 1982; Revised October 13, 1982; Accepted October 18, 1982

\begin{abstract}
The numbers of myelinated and unmyelinated fibers were counted in dorsal roots of adult rats treated neonatally with capsaicin in doses ranging from 5 to $100 \mathrm{mg} / \mathrm{kg}$. Substance $P$ and somatostatin levels in the spinal cord, dorsal roots, and sensory ganglia also were determined in control and treated animals. Capsaicin administration lead to the loss of both small myelinated and unmyelinated fibers from dorsal roots. However, whereas a near total loss, up to $94 \%$, of unmyelinated fibers was achieved after high doses of capsaicin, the reduction of myelinated fibers, even of the smallest caliber, did not exceed $40 \%$. The degree of fiber loss showed a clear dose dependency, with little detectable damage to myelinated fibers at doses of less than $50 \mathrm{mg} / \mathrm{kg}$ and with an $\mathrm{ED}_{50}$ for damage to unmyelinated fibers of 5 to $10 \mathrm{mg} / \mathrm{kg}$. In all of the structures examined, particularly the dorsal roots, a roughly parallel decrease of substance $\mathrm{P}$ and somatostatin was found with capsaicin dose. The depletions of spinal cord substance $\mathrm{P}(55 \%)$ and somatostatin $(20 \%)$ produced by neonatal capsaicin treatment were similar to those produced by dorsal rhizotomy. Capsaicin does not appear to be specific for primary afferents containing either substance P or somatostatin.
\end{abstract}

Anatomical observations of primary afferent neuron degeneration after capsaicin treatment in the neonatal rat were reported originally by Jancsó et al. (1977); see also Jancsó, 1978; Jancsó et al., 1978). Their findings have subsequently been confirmed (Nagy et al., 1980) and extended (Jancsó and Király, 1980, 1981). This degeneration, seen after a single injection of capsaicin to newborn animals, was manifest in the adult by a $70 \%$ reduction in the number of unmyelinated fibers in the saphenous nerve (Jancsó et al., 1977) and an almost complete loss of such fibers from dorsal roots (Lawson, 1981; Nagy et al., 1981). Lawson (1981) also reported significant reductions of myelinated fiber numbers in dorsal roots after capsaicin, although our own results indicated no losses of myelinated fibers from the dorsal root. Similarly, Jancsó et al. (1980) and Scadding (1980) observed large reductions in the numbers of unmyelinated fibers in peripheral nerve after capsaicin but found little or no change in the numbers of myelinated fibers.

Following the initial discovery of capsaicin-induced depletion of substance $P$ from spinal cord dorsal horn in

\footnotetext{
' We thank J. Weinheimer, M. Wynn, and J. Ditheridge for typing of the manuscript. J. I. N. is a Fellow of the Medical Research Council of Canada and M. G. is supported by a grant from Trinity College, Cambridge, United Kingdom.

${ }^{2}$ To whom requests for reprints should be sent at his current address: Department of Physiology, University of Manitoba, Winnipeg, Canada R3E 0W3.
}

adult animals by Gasparovic et al. (1964) and Jessell et al. (1978), numerous studies have shown that neonatal capsaicin treatment also leads to reduced levels of substance P (Gamse et al., 1980; Nagy et al., 1980, 1981; Helke et al., 1981; Bucsics et al., 1981; Kessler and Black, 1981) and somatostatin (Gamse et al., 1981; Nagy et al., 1981) in various nervous and non-nervous tissues known to contain primary afferent fibers. Since it has been shown that substance $P$ and somatostatin are contained in separate populations of sensory ganglia neurons (Hökfelt et al., 1976), we have sought to determine whether a selective loss of one or the other of these peptide-containing neurons could be achieved by neonatal capsaicin treatment.

Most previous studies have used a single constant dose of capsaicin. In order to explore further the specificity of the neurotoxin, the present experiments have assessed the extent of damage to primary afferent fibers after neonatal treatment with a wide range of capsaicin doses. The results fail to indicate any selectivity in the action of capsaicin on various categories of unmyelinated fibers containing different neuropeptides, and we confirm that at high doses there is also significant damage to small diameter myelinated fibers.

\section{Materials and Methods}

A total of 148 male Sprague-Dawley rats were employed in these studies, and for subsequent reference 
they are divided into the following groups according to the treatment and measurements undertaken (results of behavioral tests of pain sensitivity in these animals are described in another paper): group 1: high capsaicin dose series; animals were given $12.5,25,50,75$, and $100 \mathrm{mg} / \mathrm{kg}$ and were taken for thermal noxious threshold tests and dorsal root fiber counts $(N=38)$; group 2: low capsaicin dose series; animals were given $5,10,15,20$, and $25 \mathrm{mg}$ / $\mathrm{kg}$ and were taken for thermal, mechanical, and chemical noxious threshold tests, dorsal root unmyelinated fiber counts, and peptide measurement $(N=110)$.

Animals received either vehicle (10\% Tween-80 and $10 \%$ ethanol (v/v) in $0.9 \%$ sterile saline) or various doses of capsaicin (Sigma Chemical Co.) dissolved in vehicle subcutancously, as close to $48 \mathrm{hr}$ after birth as possible. The volumes administered ranged from 20 to $50 \mu \mathrm{l}$. One set of animals in group 2 received vehicle injections only. Immediately after injection the animals were placed near a tungsten lamp to prevent severe hypothermia, where they remained for 5 to $10 \mathrm{~min}$ completely undisturbed. Of all of the capsaicin-injected animals only two failed to survive this crucial period. Once the rat pups had recovered from the initial effects of capsaicin they were returned to their mothers, weaned at 4 weeks, and allowed, over a period of 2 to 4 months, to grow to 200 to $300 \mathrm{gm}$. By this time there was a $7.0 \%$ mortality rate among animals treated with doses of $50 \mathrm{mg} / \mathrm{kg}$ or greater. To control, to some degree, for differences between litters, a minimum of three and usually four to five littcrs were studied at any particular dose of capsaicin, controls were included in most litters, and the number of animals per litter was kept reasonably constant.

In the present study all of the animals in group 1 and some of the animals in group 2 were taken for dorsal root fiber counts, using a method similar to that described previously (Nagy et al., 1981). Animals were anesthetized with chloral hydrate and were perfused intracardially with $75 \mathrm{ml}$ of $0.1 \mathrm{M}$ phosphate buffer ( $\mathrm{pH} 7.4$ ) containing $0.1 \%$ sodium nitrate $(\mathrm{w} / \mathrm{v})$ and heparin (800 units/liter) followed by $300 \mathrm{ml}$ of a $3 \%$ glutaraldehyde $/ 3 \%$ paraformaldehyde mixture in $0.1 \mathrm{M}$ phosphate buffer ( $\mathrm{pH} 7.4$ ). The 3rd lumbar (L3) dorsal root was dissected out and postfixed overnight in the same fixative. After washing in $0.1 \mathrm{M}$ phosphate buffer ( $\mathrm{pH} 7.4$ ) containing $7.0 \%(\mathrm{w} / \mathrm{v})$ dextrose, the roots were stained for $2 \mathrm{hr}$ in $1 \%$ osmium tetroxide phosphate buffer containing $1.5 \%(\mathrm{w} / \mathrm{v})$ potassium ferrocyanide, dehydrated, and embedded in epoxy resin.

The myelinated and unmyelinated fibers in the L3 dorsal roots of all 38 animals in group 1 were analyzed. Semithin $(0.5 \mu \mathrm{m})$ transverse sections of the roots were stained with methylene blue and photographed at $\times 1000$ using Kodalith high contrast film. Photomontages of whole roots were produced at a final magnification of $\times$ 1700. From these, the total number of myelinated fibers in each root was counted: this usually ranged from 3000 to 5000 fibers. In addition, the axon circumference of about 500 to 600 myelinated fibers was measured from several randomly selected areas on the montage and converted into equivalent circle diameter, using a Reichert Videoplan image analysis system. The fibers were grouped according to diameter into 20 bins of $0.5-\mu \mathrm{m}$ increments between the diameters 0 and $10 \mu \mathrm{m}$. For each root the percentage of the 500 to 600 fibers contained in each bin was calculated, and from these percentages and the total number of myelinated fibers in that root, the total number of fibers in each bin of each root was calculated. By this procedure 38 histograms were produced of fiber numbers versus axon diameters. In order to compare the number of fibers in various size categories between control and capsaicin-treated animals, each of the histograms was normalized to reduce variability between root sizes within each group of animals. Thus, the number of fibers in each bin of a root profile was divided by the ratio of the total number of myelinated fibers in that root to the average for that particular group of animals. This procedure reduced the variability slightly but did not alter any of the conclusions obtained from calculations of absolute fiber numbers.

Unmyelinated fibers in L3 dorsal roots from animals in groups 1 and 2 were counted in 8 to 10 electron micrographs taken randomly of each root at a magnification of $\times 7000$. In control animals the total area examined typically contained between 500 and 1000 unmyelinated fibers. For the animals in group 1, the myelinated fibers in these micrographs also were counted, and from these and knowing the total number of myelinated fibers in each root, the total number of unmyelinated fibers in each root was extrapolated by simple ratio. For animals in group 2, where myelinated fibers were not counted, the total number of unmyelinated fibers in each root was calculated by multiplying the unmyelinated fiber count in each root by the ratio of the area examined in electron micrographs of the root over the total area of the root as calculated from light micrographs of each root; control values obtained by these two procedures were not significantly different.

Some of the animals in group 2 were used for determinations of substance $\mathrm{P}$ and somatostatin in various tissues. After the animals were killed by decapitation, the sciatic nerve was removed unilaterally and the lumbar $3 \mathrm{rd}, 4 \mathrm{th}, 5 \mathrm{th}$, and 6 th dorsal root ganglia, the lumbar and sacral dorsal roots, and the trigeminal ganglia were removed bilaterally. From each animal all of the dorsal roots were pooled, the lumbar ganglia were pooled, and the two trigeminal ganglia were pooled. These samples then were weighed and kept at $-70^{\circ} \mathrm{C}$ until further processing. For the extraction of tissues for radioimmunoassay of substance $\mathrm{P}$ and somatostatin, samples were placed into boiling $1 \mathrm{~m}$ acetic acid for $10 \mathrm{~min}$. The boiled samples were homogenized, boiled again for $5 \mathrm{~min}$, and allowed to stand on ice for 15 min. They then were centrifuged at $5000 \times \mathrm{g}$, the supernatant was removed, and the pellet was washed once with $1 \mathbf{M}$ acetic acid followed by recentrifugation. The original supernatant was combined with the supernatant from the wash and this was freeze-dried. The lyophilized samples were suspended in $50 \mathrm{~mm}$ sodium barbital buffer ( $\mathrm{pH} \mathrm{8.6)}$ containing $5 \%$ heat-inactivated human plasma and were centrifuged at $5000 \times g$ to eliminate insoluble material. Aliquots of this were taken for radioimmunoassay which had sensitivity limits of 70 fmol for substance $P$ and 10 fmol for somatostatin (Nagy et al., 1981).

Dorsal rhizotomy was performed on rats weighing be- 


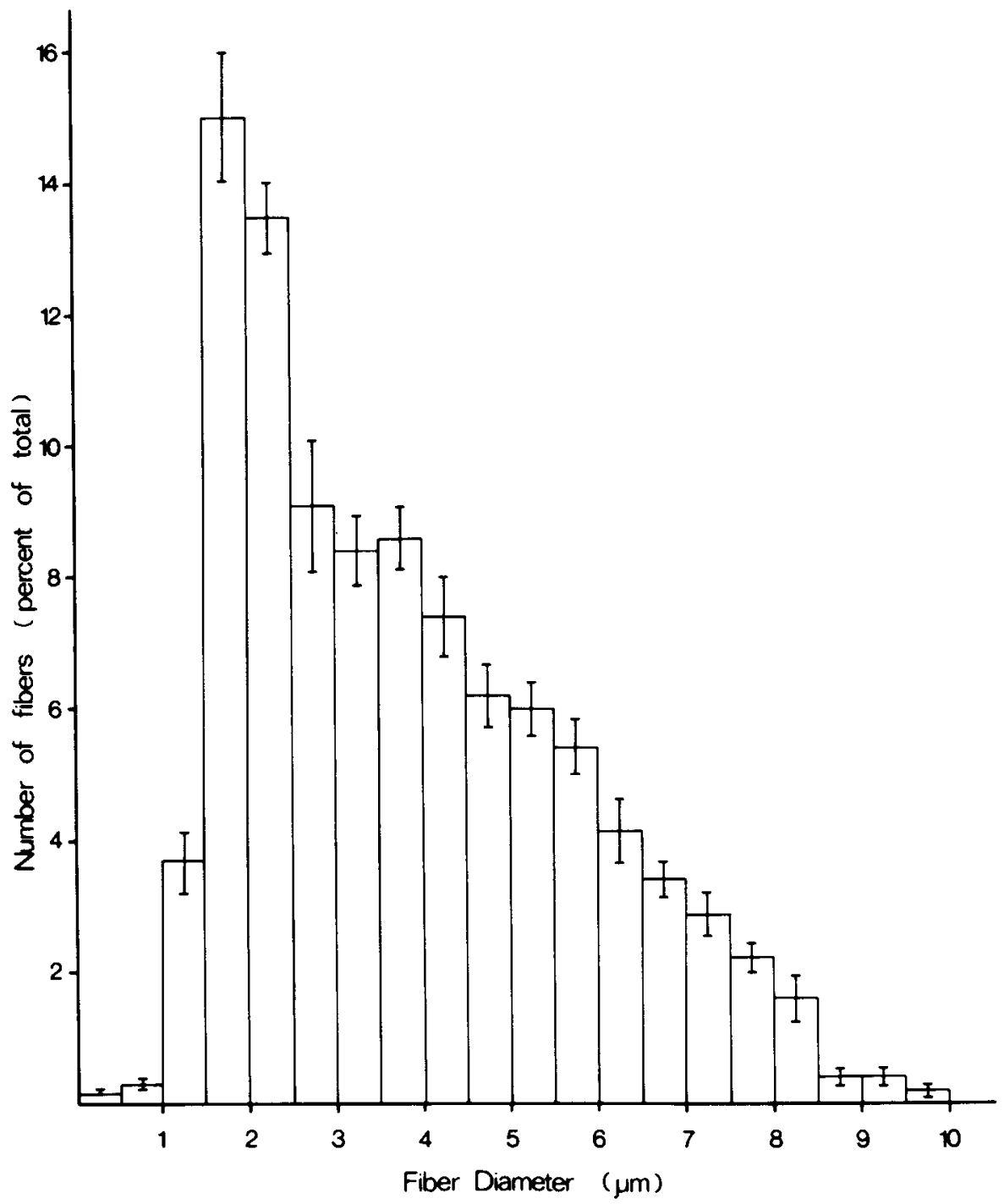

Figure 1. Histogram of the axon diameter-frequency distribution of myelinated fibers contained in the 3rd lumbar dorsal root of normal adult rats. The number of fibers in each category is expressed as the percentage of the total number of fibers contained in the roots. Fach histogram bar represents the percentage mean \pm SEM of determinations from the roots of eight animals.

TABLE I

The content of myelinated fibers of various axon diameters in the 3rd lumbar dorsal roots of normal adult animals and in roots of adult animals treated neonatally with various doses of capsaicin

The values represent the mean \pm SEM of the number of determinations shown in parentheses. Values above the stepped line drawn within the table are for the most part significantly different from controls. The data are derived from animals in group 1.

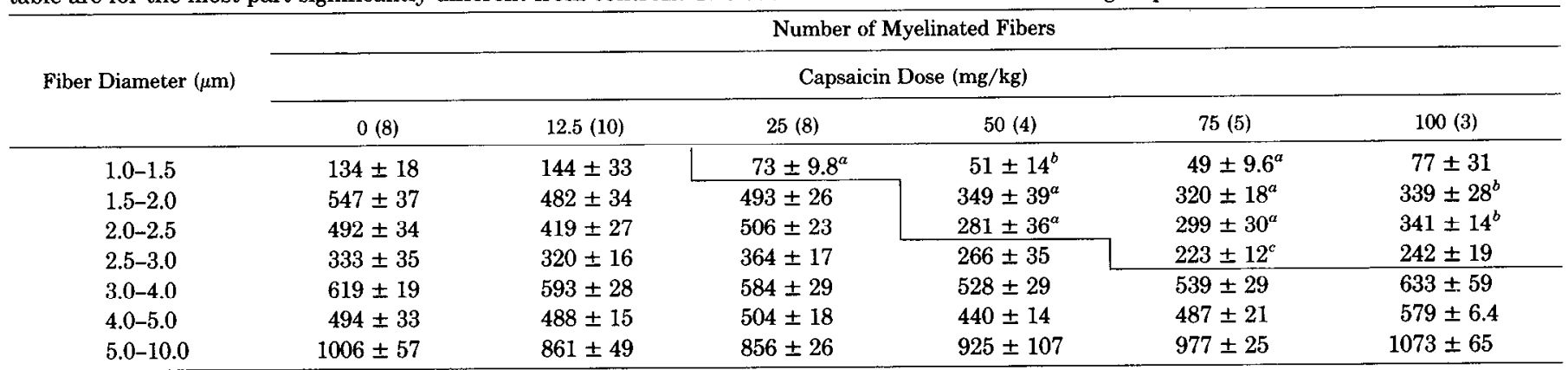

\footnotetext{
${ }^{a} p<0.01$.

${ }^{b} p<0.02$.

$p<0.05$.
} 
tween 200 and $300 \mathrm{gm}$. After a laminectomy was performed between the 2nd and 3rd lumbar sensory ganglia, all of the dorsal roots ascending in this region were transected unilaterally while care was taken to avoid bleeding and excessive trauma. This method has the advantage of denervating the lumbar enlargement while at the same time minimizing injury to this area which is some distance away from the site of the lesion. After a survival period of 10 to 14 days, the lumbar spinal cord was removed and the dorsal horn on the lesioned side was dissected from 1-mm sections of the lumbar enlargement and extracted for peptide measurements. At the same time the lesions were inspected and were found to be satisfactory. Tissues from control animals were dissected in a similar manner.

Some animals treated neonatally with $50 \mathrm{mg} / \mathrm{kg}$ of capsaicin were allowed to survive for 3 months, at which time they were used for immunohistochemical examination of substance P-containing neurons in dorsal root ganglia. Treated animals and appropriate controls were anesthetized and perfused with paraformaldehyde fixative as described elsewhere (Nagy and Hunt, 1982). The trigeminal ganglia and the 4 th and 5th lumbar dorsal root ganglia were removed and processed for substance $\mathrm{P}$ immunohistochemistry by the peroxidase-antiperoxidase technique as described previously (Nagy et al., 1981).

\section{Results}

The size distribution of myelinated fibers in the L3 dorsal roots of normal adult rats is illustrated in Figure 1. The distribution was skewed and unimodal, with axons of the $A \delta$ range (diameter $<3.0 \mu \mathrm{m}$ ) being most numerous.

The numbers of myelinated fibers of various diameters in L3 dorsal roots of control animals and after treatment of neonates with various doses of capsaicin are summarized in Table I. Fibers with axon diameters larger than $3.0 \mu \mathrm{m}$ were unaffected at any dosage of capsaicin. However, significant losses occurred among myelinated fibers of axon diameter less than $3.0 \mu \mathrm{m}$. While no myelinated fiber loss occurred after a capsaicin dose of $12.5 \mathrm{mg} / \mathrm{kg}$, at higher doses progressively larger diameter axons were lost, with an overall loss of up to $40 \%$ of $\mathrm{A} \delta$ fibers after the highest doses of capsaicin.

The effect of increasing doses of capsaicin on the number of unmyelinated fibers in L3 dorsal roots of animals in group 1 is illustrated in Figure $2 A$. There was an almost complete loss of such fibers (more than $90 \%$ ) after the highest doses of capsaicin, and the $\mathrm{ED}_{50}$ of capsaicin for damaging unmyelinated fibers was about 13 $\mathrm{mg} / \mathrm{kg}$. A similar set of results for animals in group 2, the low capsaicin dose series, is illustrated in Figure $2 B$. In this group of animals capsaicin had an $\mathrm{ED}_{50}$ of about 9 $\mathrm{mg} / \mathrm{kg}$, similar to that observed previously. No significant differences were observed in unmyelinated fiber numbers in vehicle-treated control animals.

The effects of various doses of capsaicin on substance $\mathrm{P}$ and somatostatin in dorsal roots, sciatic nerve, and sensory ganglia are illustrated in Figures 3 and 4 . These results are clearly parallel to those obtained for capsaicin-
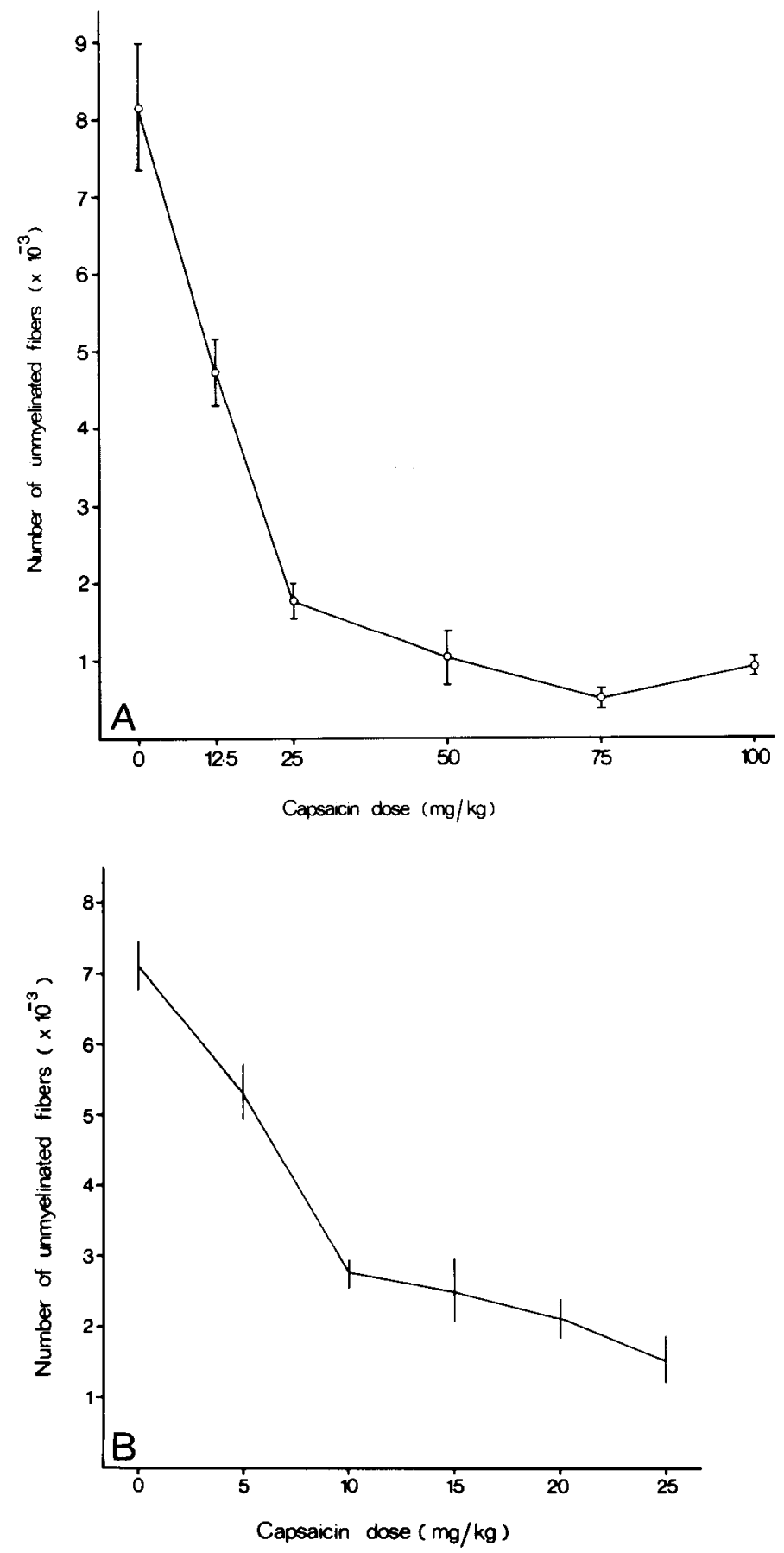

Figure 2. A, Number of unmyelinated fibers (thousands) contained in the 3rd lumbar dorsal root of adult animals after neonatal treatment with various doses of capsaicin. The values represent the mean \pm SEM of the number of animals indicated in Table I. Values at all doses of capsaicin were significantly different from controls $(p<0.001)$. The data are derived from animals in group 1. $B$, The number of unmyelinated fibers (thousands) in the 3rd lumbar dorsal roots of adult animals after neonatal treatment with various doses of capsaicin. The values represent the means \pm SEM of detcrminations from 8 control animals, 6 animals receiving $5,10,15$, and $20 \mathrm{mg} / \mathrm{kg}$, and 7 animals receiving $25 \mathrm{mg} / \mathrm{kg}$ of capsaicin. Values at all doses of capsaicin were significantly different from controls ( $p$ $<0.05$ ). The data are derived from animals in group 2 . 


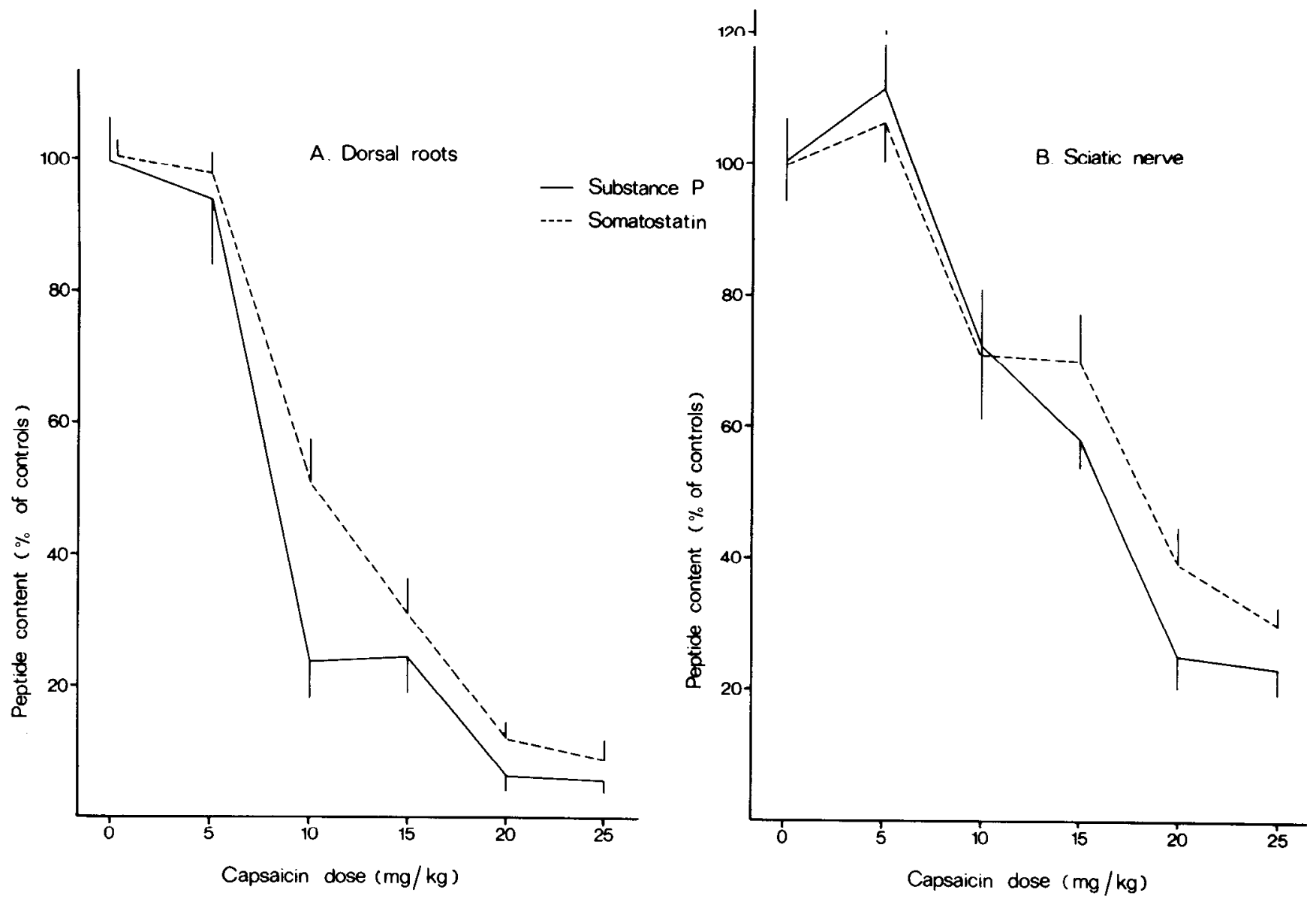

Figure 3. Substance $\mathrm{P}$ and somatostatin content in lumbar dorsal roots and sciatic nerve of adult rats after neonatal treatment with various doses of capsaicin. The values are the mean $\pm \mathrm{SEM}$ of seven to nine determinations. All values at $10 \mathrm{mg} / \mathrm{kg}$ and above are significantly different from controls $(P<0.01)$. Control levels of peptides (fmol $/ \mathrm{mg}$ of tissues) were as follows. Substance P: dorsal roots $(A), 18.2 \pm 1.3$; sciatic nerve $(B) ; 34.4 \pm 2.2$; somatostatin: dorsal roots $(A), 14.1 \pm 0.31$; sciatic nerve $(B), 11.0 \pm 0.71$.

induced loss of unmyelinated fibers in dorsal roots. There was no major difference between substance $\mathrm{P}$ and somatostatin in the extent of depletion at any of the doses of capsaicin examined. The $\mathrm{ED}_{50}$ for capsaicin-induced depletion of substance $\mathrm{P}$ or somatostatin from the various tissues examined lay between 8 and $15 \mathrm{mg} / \mathrm{kg}$. This is in reasonable agreement with the $\mathrm{ED}_{50}$ for unmyelinated fiber depletion in the L3 dorsal roots of similarly treated animals. The only suggestion of any selectivity in capsaicin action was the observation that no significant depletions of peptides from dorsal roots occurred after $5 \mathrm{mg}$ / $\mathrm{kg}$ of capsaicin, while at this dose unmyelinated fiber numbers were significantly reduced by $25 \%$ in L3 dorsal roots. The depletions of peptides from tigeminal ganglia were not as great as in the other regions examined, and they tended to level off with increasing doses of capsaicin. There were no differences in peptide content in any of the tissues in vehicle-treated animals.

The losses of substance $\mathrm{P}$ and somatostatin from the dorsal horn of the spinal cord after dorsal rhizotomy were similar to those observed previously after capsaicin (50 $\mathrm{mg} / \mathrm{kg}$ ) (Table II). However, the depletions of somatostatin in each case were considerably less than that of substance $\mathrm{P}$. If dorsal rhizotomy is taken to indicate the maximum loss of the two peptides that can be achieved after destruction of primary afferents in the dorsal horn, then the loss of these peptide-containing primary afferent systems after neonatal capsaicin treatment $(50 \mathrm{mg} / \mathrm{kg})$ would appear to be fairly complete. This conclusion is also supported by the observation that substance P-positive neurons were dramatically reduced in the 4 th and 5 th lumbar ganglia and the trigeminal ganglia of adult animals after $50 \mathrm{mg} / \mathrm{kg}$ of capsaicin neonatally (Fig. 5).

\section{Discussion}

The present results confirm our previous findings (Nagy et al., 1981) and those of Lawson and Nickels (1980) and Lawson (1981) that a virtually complete depletion of unmyelinated fibers occurs in dorsal roots of animals given capsaicin at a dosage of $50 \mathrm{mg} / \mathrm{kg}$ or greater. The somewhat smaller depletion of unmyelinated fibers seen in peripheral nerves after capsaicin (Scadding, 1980; Jancsó et al., 1980) probably is due to the presence of unaffected autonomic fibers in these nerves (Cervero and McRitchie, 1982). By examining the effects of lower doses of capsaicin, we found that the $\mathrm{ED}_{50}$ for damage to unmyelinated sensory fibers is 10 to $15 \mathrm{mg} / \mathrm{kg}$. 

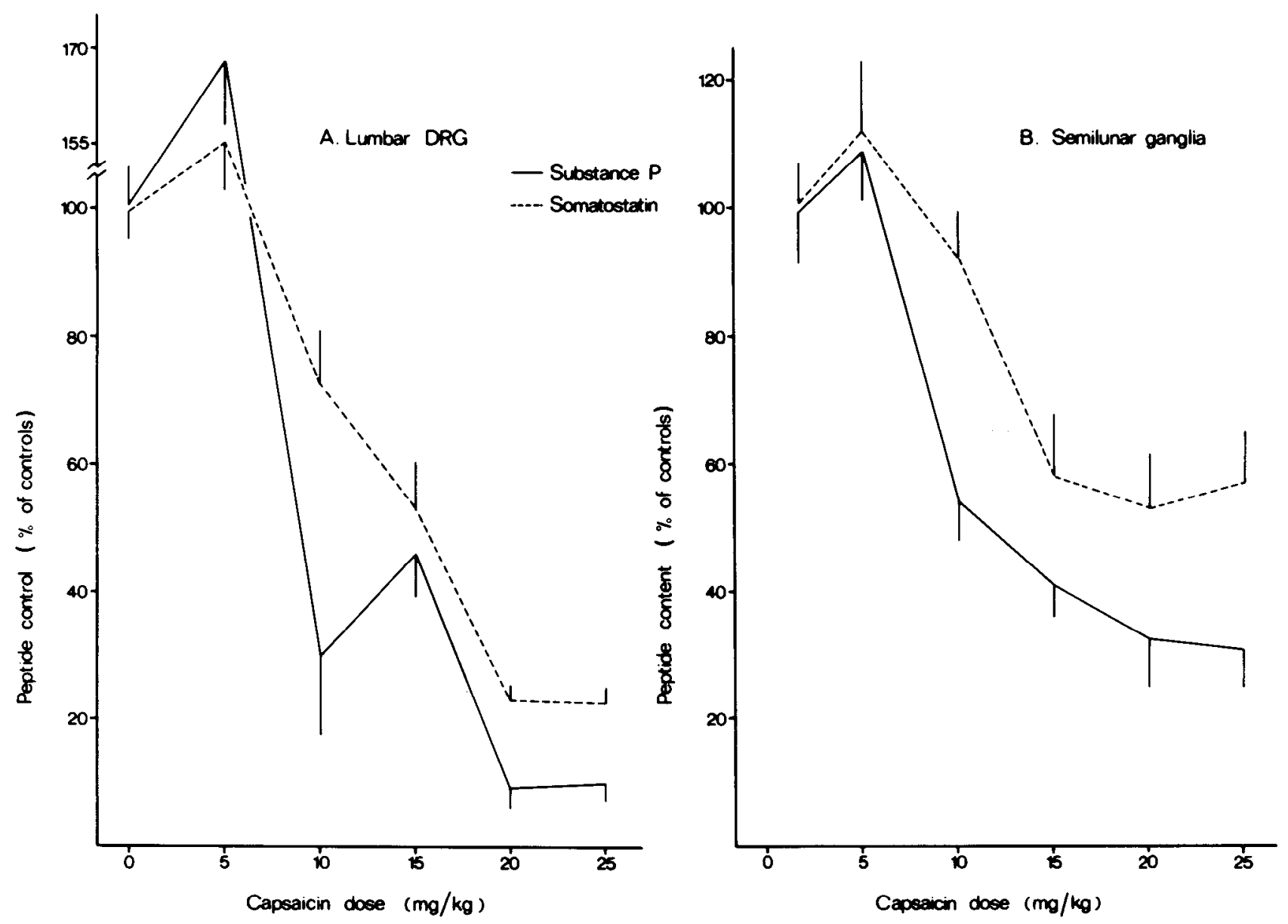

Figure 4. Substance $\mathrm{P}$ and somatostatin content in lumbar dorsal root ganglia (DRG) and trigeminal ganglia (semilunar ganglia) of adult rats after neonatal treatment with various doses of capsaicin. The values are the mean \pm SEM of seven to nine determinations. In lumbar DRG all values are significantly different from controls $(p<0.01)$ and in trigeminal ganglia values for substance $\mathrm{P}$ are significantly different from controls at $10 \mathrm{mg} / \mathrm{kg}$ and above and for somatostatin at $15 \mathrm{mg} / \mathrm{kg}$ and above $(p<$ 0.01). Control levels of peptides (fmol/mg tissue) were as follows. Substance P: lumbar DRG, $18.3 \pm 1.9$; trigeminal ganglia, 73.5 \pm 5.9 ; somatostatin: lumbar DRG, $16.8 \pm 0.8$; trigeminal ganglia, $15.5 \pm 1.0$.

TABLE II

The effect of neonatal capsaicin treatment and dorsal rhizotomy on substance $P$ and somatostatin in the dorsal horn of the lumbar spinal cord

Peptide levels were determined 3 months after neonatal capsaicin treatment and 10 to 14 days after dorsal rhizotomy. All values are the mean \pm SEM of six to eight determinations and are significantly different from controls $(p<0.05)$. Absolute dorsal horn content of substance $P$ was $347 \pm 19 \mathrm{fmol} / \mathrm{mg}$ of tissue and of somatostatin was $222 \pm 13 \mathrm{fmol} / \mathrm{mg}$ of tissue.

\begin{tabular}{|c|c|c|}
\hline & Substance $P$ & Somatostatin \\
\hline & \multicolumn{2}{|c|}{$\%$ of control } \\
\hline Neonatal capsaicin ${ }^{a}$ & $44.5 \pm 5.6$ & $80.5 \pm 7.7$ \\
\hline Dorsal rhizotomy & $52.8 \pm 8.7$ & $72.0 \pm 7.9$ \\
\hline
\end{tabular}

${ }^{a}$ The data are from Nagy et al. (1981).

Higher doses of capsaicin are needed before any detectable damage occurs to myelinated fibers. The failure to detect any depletion of these in peripheral nerves (Scadding, 1980; Janscó et al., 1980) probably is related to the relatively small depletion of these fibers even after the highest doses of capsaicin. Lawson (1981) reported reductions in total myelinated fiber numbers of $24 \%$ and $34 \%$ in thoracic and lumbar dorsal roots, respectively, after a dose of capsaicin that was approximately $70 \mathrm{mg} /$ $\mathrm{kg}$. The depletion of about $25 \%$ in the total number of myelinated fibers in L3 roots observed here after capsaicin at doses of $50 \mathrm{mg} / \mathrm{kg}$ or greater is in good agreement with this. Surprisingly, in a previous study we failed to observe any significant loss in myelinated fibers from dorsal roots of animals given $50 \mathrm{mg} / \mathrm{kg}$ of capsaicin. Since this is just above the dose level needed to damage myelinated fibers, the discrepancy between these two studies probably reflects minor differences in experimental procedure that we cannot account for.

Several important points arise regarding the loss of myelinated fibers in dorsal roots. First, only the A $\delta$ fibers were affected, and among these, progressively larger fibers were depleted with increasing doses of capsaicin. In addition, there appeared to be a ceiling effect of neurotoxicity for any particular caliber of fibers. Once a certain level of depletion was attained, higher doses had little or no further effect on the remaining fibers. This suggests a greater susceptibility to capsaicin in a subpopulation of fibers contained in the 0 - to $3-\mu \mathrm{m}$ caliber range. Alter- 

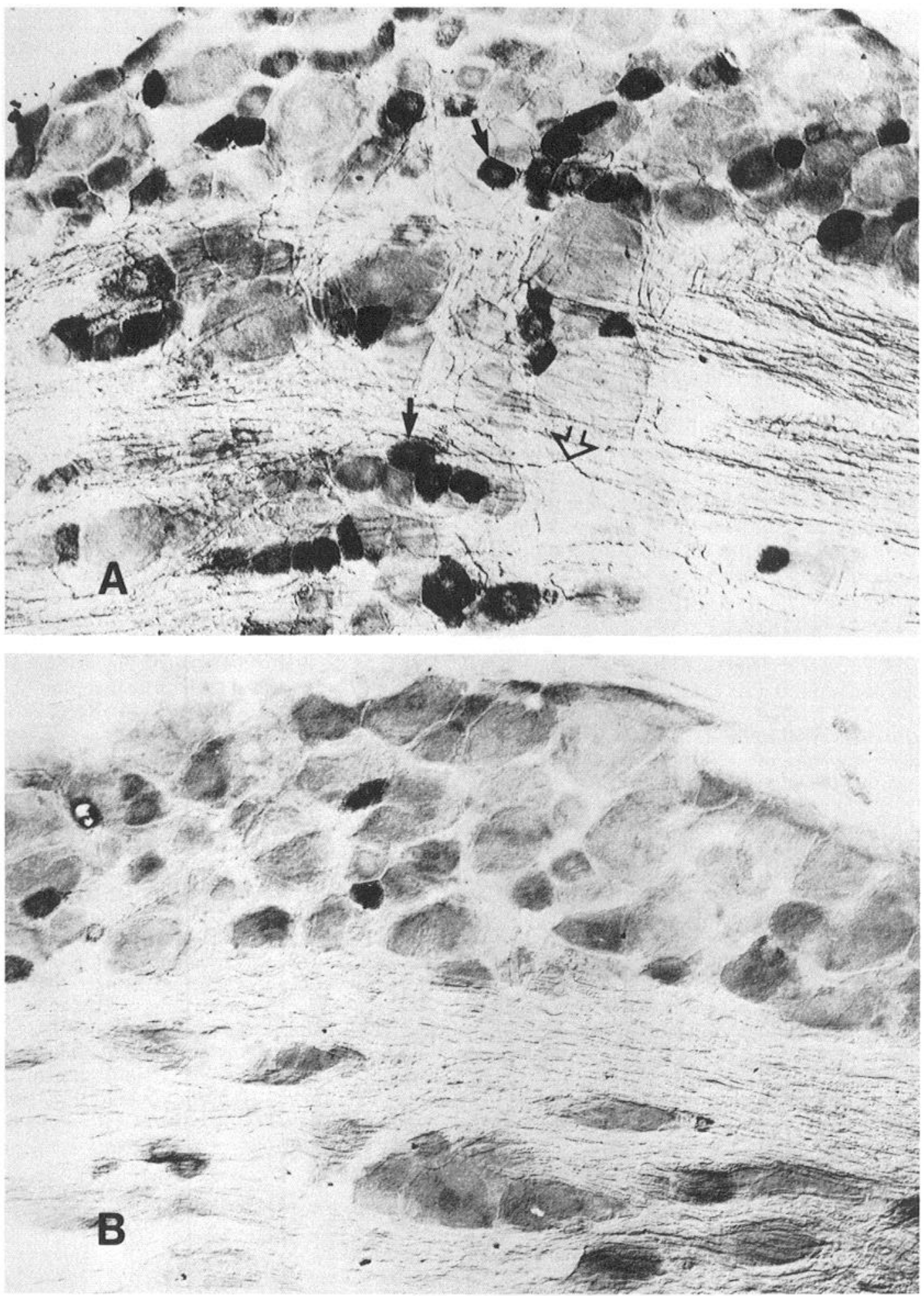

Figure 5. Sections of the 4th lumbar ganglia stained immunohistochemically for substance P. $A$, Normal adult animals; $B$, adult animals treated neonatally with $50 \mathrm{mg} / \mathrm{kg}$ of capsaicin. In $A$, the solid arrows indicate substance P-positive neurons and the open arrow indicates substance P-stained fibers. In $B$, both types of staining are largely absent. Magnification $\times 270$.

natively, depletions of A $\delta$ fibers greater than those observed may not be conducive to survival of the animal. Clearly, capsaicin causes the depletion of both myelinated and unmyelinated fibers from dorsal roots, but doses of capsaicin can be chosen which lead to selective damage of unmyelinated fibers in dorsal roots.

There seems to be little possibility, however, of a differential neurotoxic effect of neonatal capsaicin treatment on substance P- or somatostatin-containing dorsal root ganglia neurons. The parallel depletions of substance 
P- and somatostatin-containing sensory neurons with increasing capsaicin doses clearly indicate a lack of neurotoxic selectivity of capsaicin for either of these sensory neuron populations. Only at the lowest dose of capsaicin tested $(5 \mathrm{mg} / \mathrm{kg}$ ) was there any indication of a possible selective neurotoxic action on unmyelinated fibers nol containing substance $\mathrm{P}$ or somatostatin.

The present results also throw further light on the localization of neuropeptides in the dorsal horn. We previously reported that capsaicin $(50 \mathrm{mg} / \mathrm{kg})$ given neonatally led to almost total depletion of substance $\mathrm{P}$ and somatostatin from dorsal roots (Nagy et al., 1981). Yet, this led to a maximum loss of substance $\mathrm{P}$ from the dorsal horn of about $55 \%$ and a somatostatin loss of about $20 \%$. The present results show that denervation of the lumbar dorsal horn by dorsal rhizotomy leads to depletions of somatostatin and substance $\mathrm{P}$ from the dorsal horn similar to those seen after capsaicin. Although adaptive changes may take place after rhizotomy or capsaicin treatment, these results suggest that primary afferent fibers and terminals account for about $50 \%$ of the substance $\mathrm{P}$ content of rat dorsal horn, but only about $20 \%$ of the somatostatin.

\section{References}

Bucsics, A., A. Saria, and F. Lembeck (1981) Substance P in the adrenal gland: Origin and species distribution. Neuropeptides 1: 329-341.

Cervero, F., and H. A. McRitchie (1982) Neonatal capsaicin does not affect efferent fibers of the autonomic nervous system: Functional evidence. Brain Res. 239: 283-288.

Gamse, R., P. Holzer, and F. Lembeck (1980) Decrease of substance $\mathrm{P}$ in primary afferent neurons and impairment of neurogenic plasma extravasation by capsaicin. Br. J. Pharmacol. 68: 207-213.

Gamse, R., S. E. Leeman, P. Holzer, and F. Lembeck (1981) Differential effects of capsaicin on the content of somatostatin, substance $P$, and neurotensin in the nervous system of the rat. Naunyn Schmiedebergs Arch. Pharmacol. 317: 140148.

Gasparovic, I., S. Hadzovic, S. Hukovic, and P. Stern (1964) Contribution to the theory that substance $\mathrm{P}$ has a transmitter role in sensitive pathway. Med. Exp. 10: 303-306.

Helke, C. J., J. A. DiMicco, D. M. Jacobowitz, and I. J. Kopin (1981) Effects of capsaicin administration to neonatal rats on the substance $\mathrm{P}$ content of discrete CNS regions. Brain Res. 222: 428-431.

Hökfelt, T., R. Elde, O. Johansson, R. Luft, G. Nilsson, and A.
Arimura (1976) Immunohistochemical evidence for separate populations of somatostatin and substance $\mathrm{P}$ containing primary neurons in the rat. Neuroscience 1: 131-136.

Jancsó, G. (1978) Selective degeneration of chemosensitive primary sensory neurons induced by capsaicin: Glial changes. Cell Tissue Res. 195: 145-152.

Jancsó, G., and E. Király (1980) Distribution of chemosensitive primary sensory afferents in the central nervous system of the rat. J. Comp. Neurol. 190: 781-792.

Jancsó, G., and E. Király (1981) Sensory neurotoxins: Chemically induced selective destruction of primary sensory neurons. Brain Res. 210: 83-89.

Jancsó, G., E. Király, and A. Janscó-Gábor (1977) Pharmacologically induced selective degeneration of chemosensitive primary sensory neurons. Nature 270: 741-743.

Jancsó, G., G. Savay, and E. Király (1978) Appearance of histochemically detectable ionic calcium in degenerating primary sensory neurons. Acta Histochem. (Jena) 62: 165-169.

Jancsó, G., E. Király, and A. Jancsó-Gábor (1980) Chemosensitive pain fibers and inflammation. Int. J. Tissue React. 2: $57-66$.

Jessell, T., L. L. Iversen, and A. C. Cuello (1978) Capsaicininduced depletion of substance $\mathbf{P}$ from primary sensory neurones. Brain Res. 152: 183-188.

Kessler, J. A., and I. B. Black (1981) Similarities in development of substance $P$ and somatostatin in peripheral sensory neurons: Effect of capsaicin and nerve growth factor. Proc. Natl. Acad. Sci. U. S. A. 78: 4644-4647.

Lawson, S. N. (1981) Dorsal root ganglion neurons and dorsal roots: Effect of neonatal capsaicin. In Spinal Cord Sensation: Sensory Processing in the Dorsal Horn, A. G. Brown, ed., pp. 57-58, Scottish Press, Ldinburgh.

Lawson, S. N., and S. M. Nickels (1980). The use of morphometric techniques to analyse the effect of neonatal capsaicin treatment on dorsal root ganglia and dorsal roots. J. Physiol. (Lond.) 303: 12P.

Nagy, J. I., and S. P.Hunt (1982) Fluoride-resistant acid phosphatase-containing neurons in dorsal root ganglia are separate from those containing substance $\mathrm{P}$ or somatostatin. Neuroscience 7: 89-97.

Nagy, J. I., S. R. Vincent, W. A. Staines, H. C. Fibiger, T. D. Reisine, and H. I. Yamamura (1980) Neurotoxic action of capsaicin on spinal substance P neurons. Brain Res. 186: 435444.

Nagy, J. I., S. P. Hunt, L. L. Iversen, and P. C. Emson (1981) Biochemical and anatomical observations on the degeneration of peptide-containing primary afferent neurons after neonatal capsaicin. Neuroscience 10: 1923-1934.

Scadding, J. W. (1980) The permanent anatomical effects of neonatal capsaicin on somatosensory nerves. J. Anat. 131: $473-484$. 